\title{
Development of bisexual attractants for diamondback moth, Plutella xylostella (Lepidoptera: Plutellidae) based on sex pheromone and host volatiles
}

\author{
Jianqing DAI, ${ }^{1,2, \dagger}$ Jianyu DENG ${ }^{3}$ and Jiawei Du ${ }^{1, *}$ \\ ${ }^{1}$ Institute of Plant Physiology \& Ecology, Shanghai Institutes for Biological Sciences, Chinese Academy of Sciences, Graduate \\ School of the Chinese Academy of Sciences; Shanghai 200032, China \\ ${ }^{2}$ Guangdong Entomological Institute, Guangdong Academy of Sciences; Guangzhou 510260, China \\ ${ }^{3}$ School of Agriculture and Food Science, Zhejiang Forestry University; Hangzhou 311300, China
}

(Received 23 April 2008; Accepted 8 August 2008)

\begin{abstract}
Males and females of the diamondback moth (DBM), Plutella xylostella (L.) were investigated for electroantennogram responses to 13 volatiles from Brassicaceae in the laboratory, and showed stronger responses to benzaldehyde, phenylacetaldehyde, allyl isothiocyanate, $(Z)$-3-hexen-1-ol and $(Z)-3$-hexenyl acetate than to monoterpenic volatiles. In field trapping experiments, traps baited with the synthetic female sex pheromone of DBM $+(Z)-3$-hexenyl acetate or pheromone $+(Z)$-3-hexen-1-ol significantly enhanced male catches in three brassicaceous crops, compared with traps baited with synthetic sex pheromone alone. Traps baited with pheromone $+(Z)-3$-hexenyl acetate and pheromone $+(Z)$-3-hexenyl acetate $+(Z)$-3-hexen-1-ol+allyl isothiocyanate enhanced male catches by $46.4 \%$ and $75.0 \%$, respectively, compared with synthetic sex pheromone alone, and caught an average of $15.8 \pm 2.2$ and $18.6 \pm 2.7$ females/trap/day, respectively, during the whole trapping period. Results indicate that traps baited with pheromone $+(Z)$-3-hexenyl acetate $+(Z)$-3-hexen-1-ol+allyl isothiocyanate have a stronger attraction to both male and female DBM and may provide more effective tools for monitoring and controlling this insect pest.
\end{abstract}

Key words: Plutella xylostella; sex pheromone; kairomone; electroantennogram; field trapping

\section{INTRODUCTION}

The diamondback moth (DBM), Plutella $x y$ lostella (L.) (Lepidoptera: Plutellidae) is one of the most important pests of cruciferous crops throughout the world (Talekar and Shelton, 1993). DBM feeds on all brassicaceous crop plants, cole crops, and several greenhouse plants, particularly cabbage and other cruciferous plants of the genus Brassica (Reddy and Guerrero, 2004). One of the factors that have enabled $P$. xylostella to cause so much damage is its ability to develop resistance rapidly to many classes of insecticides (Talekar and Shelton, 1993). This has prompted an evaluation of alternative pest management strategies including applications of its sex pheromone.

The major female sex pheromone components of DBM are (Z)-11-hexadecenal (Z11-16:Ald), (Z)- 11-hexadecenyl acetate (Z11-16:Ac), and (Z)-11hexadecenol (Z11-16:OH) (Chow et al., 1977; Tamaki et al., 1977; Koshihara et al., 1978; Koshihara and Yamada, 1980). The synthetic sex pheromone has been utilized to monitor and control DBM (Môttus et al., 1997; Reddy and Guerrero, 2000a). In China, more farmers prefer mass trapping to mating disruption with sex pheromone lures for control of insect pests because it is convenient and inexpensive. However, field tests of mass trapping did not result in high efficiency in the control of DBM, and it has often been deemed to be less competitive than other means of control during the past years $(\mathrm{Du}, 2000)$. It seems likely that the best way to improve the efficiency of the mass trapping technique is to study and develop a highly efficient lure for both male and female DBM.

\footnotetext{
* To whom correspondence should be addressed at: E-mail: jqdai@sibs.ac.cn

${ }^{\dagger}$ Present address: Guangdong Entomological Institute, Guangdong Academy of Sciences, Guangzhou 510260, China. DOI: $10.1303 / \mathrm{aez} .2008 .631$
} 
Previous studies have demonstrated that chemicals from host plants often enhance insect responses to sex pheromones (Landolt and Phillips, 1997; Reddy and Guerrero, 2004). Host odor enhancement of attraction responses of males to pheromones was reported in several insect orders (Dickens et al., 1993; Light et al., 1993; Fang and Zhang, 2002; Deng et al., 2004). Reddy and Guerrero $(2000 \mathrm{~b})$ reported that the mixture of $(Z)-3$ hexenyl acetate with synthetic sex pheromone had a synergistic effect on the capture of $P$. xylostella moths in the field in comparison with synthetic sex pheromone alone. Some volatile compounds from Brassicaceae have been identified and proven attractive to adult DBM (Reddy and Guerrero, 2000b; Han et al., 2001). Here we selected several volatile compounds reported to be produced by host plants of DBM (Reddy and Guerrero, 2000b; Han et al., 2001). We then tested the electroantennogram (EAG) responses elicited by them in the laboratory. The trapping efficiency of these plant volatiles combined with synthetic sex pheromone for both males and females of DBM was assessed in the field. Based on these studies, we developed effective attractants for simultaneous trapping of both sexes of DBM to improve mass trapping efficiency in the fields.

\section{MATERIALS AND METHODS}

Insects and plants. P. xylostella adults were collected from cabbage (Brassica oleracea var. capitata Linn.) fields in Songjiang $\left(31.1^{\circ} \mathrm{N}, 121.4^{\circ} \mathrm{E}\right)$, Shanghai, China in 2002, and reared in the laboratory on potted Chinese cabbage (B. rapa cv. gr. Pekinensis) for two generations before use in experiments. The laboratory was maintained at $25 \pm 2{ }^{\circ} \mathrm{C}$ and $60 \pm 10 \% \mathrm{RH}$, under a reversed $14 \mathrm{~L}$ : $10 \mathrm{D}$ photoperiod. Male and female DBM larvae were separated as described by Liu and Tabashnik (1997). Newly emerged adults were provided with cotton balls saturated with $10 \%$ sucrose solution.

Chemicals. (Z)-11-Hexadecenal (Z11-16:Ald), $(Z)$-11-hexadecenyl acetate (Z11-16:Ac) and (Z)11-hexadecenol (Z11-16:OH) were obtained commercially (Shin-Etsu Chemical Industries, Japan). (Z)-3-Hexenyl acetate was synthesized and purified by column chromatography in our laboratory (Wang, 1987). The purity of these compounds was measured using gas chromatography, and the sam- ple was found to be more than $95 \%$ pure. Hexanal (purity $\geq 98 \%$ ), 1-hexen-3-ol (purity $\geq 98 \%$ ), 1octen-3-ol (purity $\geq 98 \%$ ), $(E)$-2-hexenal (purity $\geq 99 \%$ ), (Z)-3-hexen-1-ol (purity $\geq 98 \%$ ), ( \pm )linalool (purity $\geq 98 \%$ ), $R-(+)$-limonene (purity $\geq 97 \%$ ), and $\alpha$-pinene (a blend of the enantiomers of $R$ - $(+)-\alpha$-pinene and $S$-(-)- $\alpha$-pinene) (purity $\geq 97 \%$ ) were purchased from Sigma-Aldrich Chemical Co. Ltd. Allyl isothiocyanate (purity $\geq 94 \%$ ), benzaldehyde (purity $\geq 98 \%$ ), phenylacetaldehyde (purity $\geq 94 \%$ ), benzoic acid (purity $\geq 94 \%$ ), hexane (purity $\geq 99 \%$ ) were purchased from the Chinese Medical Chemical Company, Shanghai. Peanut oil was bought in a Shanghai supermarket. Plastic centrifugal tubes were obtained from the Shanghai Biological Engineering Co., Ltd.

Electroantennogram (EAG) responses. EAG responses from isolated male and female antennae were recorded according to the method described by Fang and Zhang (2002). An antenna of a 3-dayold moth was excised from its base with microscissors. The base of the isolated antenna was connected to the indifferent electrode, while the tip, from which a few distal segments were cut in order to get better contact, was connected to the recording electrode. EAG signals were amplified and monitored with a head-stage preamplifier and further amplified and processed with a PC-based signal processing system $\left(\right.$ Syntech $^{\circledR}$, The Netherlands).

EAG evaluations were conducted to screen for effective compounds, and 13 volatile components, including benzaldehyde, phenylacetaldehyde, allyl isothiocyanate, $(Z)$-3-hexen-1-ol, $(Z)$-3-hexenyl acetate, 1-hexen-3-ol, (E)-2-hexenal, 1-octen-3-ol, hexanal, benzoic acid, linalool, limonene and $\alpha$ pinene, were evaluated. Test compounds were dissolved in analytical grade hexane. The test concentration of each solution was $4 \mu \mathrm{g} / \mu \mathrm{l}$. Five microlitres of each solution was applied to a piece of filter paper $(5 \times 30 \mathrm{~mm})$, and the solvent was allowed to evaporate for $20 \mathrm{~s}$ before the filter paper was inserted into a glass Pasteur pipette $(15 \mathrm{~cm}$ long). The tip of the pipette was inserted into a small hole $(3 \mathrm{~mm}$ in diameter, $12 \mathrm{~cm}$ from the outlet) of a main airflow tube $(12 \mathrm{~mm}$ in diameter, 17 $\mathrm{cm}$ long) in which a continuous, charcoal filtered and moistened airflow $(4 \mathrm{ml} / \mathrm{s})$ was blown through onto the prepared antenna. A $0.5 \mathrm{~s}$ puff of charcoal- 
filtered airflow $(4 \mathrm{ml} / \mathrm{s})$ was introduced through the large end of the Pasteur pipette, transporting the test chemicals to antenna for stimulation, using an electronically controlled stimulus flow controller $\left(\mathrm{CS} 05\right.$, Syntech $\left.{ }^{\circledR}\right)$. Intervals between stimuli were at least $60 \mathrm{~s}$. Each chemical was tested with a hexane blank presented before and after with 1-min intervals between tests. Each compound was tested with ten moth antennae, and each antenna was tested three times.

To account for solvent and other background effects, we subtracted the averaged EAG responses to hexane recorded before and after each test material as described by Dickens (1984). EAG responses for all test chemicals were standardized to the absolute EAG response of $20 \mu \mathrm{g}$ of benzaldehyde per filter paper strip, which was set to 1 . For statistical analyses, data were arcsine square-root transformed to meet the assumption of normality and homogeneity of variances. The significance of differences $(p<0.05)$ in the intensity of EAG response to test chemicals was assessed using analysis of variance (ANOVA) and means compared using Duncan's multiple range test (Duncan, 1955).

\section{Field trapping.}

Trapping efficiency of a single plant volatile. To assess the trapping efficiency of a single plant volatile, field trials were conducted in cabbage fields at the Songjiang Agricultural Experiment Station (Songjiang District, Shanghai, China) from October to December, 2002. Treatments were (i) synthetic sex pheromone $(\mathrm{Ph})$, (ii) $(Z)$-3-hexenyl acetate, (iii) allyl isothiocyanate, (iv) (Z)-3-hexen1-ol, (v) benzaldehyde, (vi) phenylacetaldehyde, and (vii) control.

Trapping efficiency of synthetic sex pheromone combined with a single plant volatile. To assess the trapping efficiency of synthetic sex pheromone combined with a single plant volatile, field trials were conducted in cabbage and cauliflower fields at Songjiang Agricultural Experiment Station from May to July of 2003 and 2004, and in a Chinese cabbage field at Pudong Agriculture Farm (Pudong District, Shanghai, China) from October to December, 2003. Treatments were (i) $\mathrm{Ph}$, (ii) $\mathrm{Ph}+(Z)-3$ hexenyl acetate, (iii) $\mathrm{Ph}+$ allyl isothiocyanate, (iv) $\mathrm{Ph}+(Z)$-3-hexen-1-ol, (v) $\mathrm{Ph}+$ benzaldehyde, (vi) $\mathrm{Ph}+$ phenylacetaldehyde, and (vii) control.

Trapping efficiency of synthetic sex pheromone combined with mixed volatiles. Field trials were also conducted in cabbage fields at Songjiang Agricultural Experiment Station from October to December, 2004, May to July and October to December, 2005 to evaluate the trapping efficiency of synthetic sex pheromone combined with mixed volatiles. Treatments were (i) $\mathrm{Ph}$, (ii) $\mathrm{Ph}+(Z)-3$ hexenyl acetate, (iii) $\mathrm{Ph}+(Z)-3$-hexenyl acetate + allyl isothiocyanate, (iv) $\mathrm{Ph}+(Z)-3$-hexen-1-ol+ allyl isothiocyanate, (v) $\mathrm{Ph}+(Z)$-3-hexenyl acetate $+(Z)-3$-hexen-1-ol+allyl isothiocyanate, and (vi) control. All field trials were conducted during the peak emergence period of adult $P$. xylostella from May to July and from October to December every year in Shanghai, China.

Two types of dispensers were used in the traps during field trapping. One was a red rubber septum impregnated with $50 \mu \mathrm{g}$ of synthetic sex pheromone (a mixture of Z11-16:Ald, Z11-16:Ac and $Z 11-16: \mathrm{OH}$ at a ratio of $7: 3: 1$ ) for all experiments, and the second was a $600 \mu 1$ plastic centrifugal tube loaded with test chemicals in $10 \mu \mathrm{l}$ of peanut oil. A $1 \mathrm{~mm}$ hole was bored in the cap of the tube when the trap was mounted in the field (Deng et al., 2004). Yellow plastic water basins (20 $\mathrm{cm}$ in diameter, $10 \mathrm{~cm}$ in height) were used as traps in field trapping.

The two different dispensers were hung close together $2 \mathrm{~cm}$ above the water surface of the traps. The traps were placed about $20 \mathrm{~cm}$ above the plant canopy. The layout of the trial followed a completely randomized block design. The distance between traps was about $20 \mathrm{~m}$. Traps were rotated every three days to minimize the effects of trap location, and lures were replaced every 10 days. Insects captured in the traps were removed every two days, counted, sexed and cataloged. The trap with a plastic centrifugal tube containing $10 \mu \mathrm{l}$ of peanut oil was used as a control. Four or six replicates were used for each treatment. Over the three years that experiments were conducted, plots were conventionally tilled with fertilizers and herbicides applied according to current recommendations from the Plant Protection Station. Each plot was separated from other crucifers by at least $50 \mathrm{~m}$.

Data analysis. Before analysis, counts were transformed to $\log (x+1)$ in order to homogenize variances and standardize means and then were subjected to one-way analysis of variance (ANOVA), and when significant treatment differences were indicated, Duncan's multiple range test 
(DMRT) $(p<0.05$ is assumed unless stated differently) was used to separate mean trap captures. Untransformed means ( $\pm \mathrm{SE})$ are given in tests, whereas statistical results refer to transformed data. Statistical analyses were performed using the SPSS 11.0 for Windows statistical package (SPSS, IL, USA).

\section{RESULTS}

\section{EAG responses}

The EAG results showed that all volatile components tested could elicit electroantennogram (EAG) responses from male and female DBM (males: $F_{11,119}=19.58, p<0.0001$; females: $F_{11,119}=26.36$, $p<0.0001$ ) (Table 1), Of these volatiles, benzaldehyde, phenylacetaldehyde, allyl isothiocyanate, (Z)-3-hexen-1-ol and (Z)-3-hexenyl acetate elicited stronger EAG responses, and the EAG responses elicited by monoterpenic volatiles were weaker (Table 1).

\section{Field trapping}

\section{Trapping efficiency of a single plant volatile}

Traps baited with a single plant volatile compound or synthetic sex pheromone $(\mathrm{Ph})$ alone captured significantly more DBM males than the control (males: $F_{6,27}=154.76, p<0.0001$; females: $\left.F_{6,27}=42.13, p<0.0001\right)$. Traps baited with $(Z)$ 3-hexenyl acetate, (Z)-3-hexen-1-ol and sex pheromone alone captured significantly more males than traps with allyl isothiocyanate, benzaldehyde and phenylacetaldehyde. Fewer males were caught in the trap baited with a single plant volatile compound than with the sex pheromone (Duncan, $p<0.05$ ) (Table 2). However, significantly more females were found in the traps baited with allyl isothiocyanate $(4.6 \pm 0.3$ females/trap/ day) or $(Z)$-3-hexenyl acetate $(3.6 \pm 0.4$ females/ trap/day) alone, as compared to sex pheromone traps (Table 2).

Trapping efficiency of synthetic sex pheromone combined with a single plant volatile

Traps baited with synthetic sex pheromone $(\mathrm{Ph})+(Z)$-3-hexenyl acetate and $\mathrm{Ph}+(Z)$-3-hexen1-ol captured significantly more males than traps with $\mathrm{Ph}$ alone. However, there were no significant differences between treatments of $\mathrm{Ph}+$ allyl isothiocyanate, $\mathrm{Ph}+$ benzaldehyde, $\mathrm{Ph}+$ phenylacetaldehyde and $\mathrm{Ph}$ alone (cabbage field, males:
Table 1. Electroantennogram (EAG) responses of DBM elicited by volatile compounds of brassicaceous crops

\begin{tabular}{|c|c|c|c|}
\hline \multirow{2}{*}{ No. } & \multirow{2}{*}{ Compounds } & \multicolumn{2}{|c|}{$\begin{array}{l}\text { Relative EAG values } \\
\qquad(\text { mean } \pm \mathrm{SE})^{\mathrm{a}}\end{array}$} \\
\hline & & Male & Female \\
\hline 1 & Benzaldehyde & 1.00 & 1.00 \\
\hline 2 & Phenylacetaldehyde & $0.93 \pm 0.12 \mathrm{~d}$ & $0.97 \pm 0.05 \mathrm{c}$ \\
\hline 3 & Allyl isothiocyanate & $0.77 \pm 0.07 \mathrm{~cd}$ & $0.81 \pm 0.07 \mathrm{c}$ \\
\hline 4 & (Z)-3-Hexen-1-ol & $0.72 \pm 0.09 \mathrm{~cd}$ & $0.46 \pm 0.09 \mathrm{~b}$ \\
\hline 5 & (Z)-3-Hexenyl acetate & $0.67 \pm 0.08 \mathrm{bcd}$ & $0.44 \pm 0.05 b$ \\
\hline 6 & 1-Hexen-3-ol & $0.56 \pm 0.06 \mathrm{bc}$ & $0.49 \pm 0.11 b$ \\
\hline 7 & (E)-2-Hexenal & $0.45 \pm 0.05 \mathrm{ab}$ & $0.26 \pm 0.06 \mathrm{a}$ \\
\hline 8 & 1-Octen-3-ol & $0.43 \pm 0.07 \mathrm{ab}$ & $0.27 \pm 0.05 \mathrm{a}$ \\
\hline 9 & Hexanal & $0.32 \pm 0.04 \mathrm{a}$ & $0.21 \pm 0.03 \mathrm{a}$ \\
\hline 10 & Benzoic acid & $0.31 \pm 0.05 \mathrm{a}$ & $0.23 \pm 0.04 \mathrm{a}$ \\
\hline 11 & Linalool & $0.28 \pm 0.02 \mathrm{a}$ & $0.25 \pm 0.06 \mathrm{a}$ \\
\hline 12 & Limonene & $0.26 \pm 0.03 \mathrm{a}$ & $0.21 \pm 0.04 \mathrm{a}$ \\
\hline 13 & $\alpha$-Pinene & $0.23 \pm 0.07 \mathrm{a}$ & $0.22 \pm 0.03 \mathrm{a}$ \\
\hline
\end{tabular}

${ }^{\text {a }}$ Data within the same column followed by different letters are significantly different ( $p<0.05$ by Duncan's multiple range test). Mean EAGs (relative values) \pm standard errors $(\mathrm{mV}) ; n=10$ per treatment.

Table 2. Average daily catches of male and female DBM by traps baited with a single plant volatile in cabbage fields ${ }^{\mathrm{a}}$

\begin{tabular}{|c|c|c|}
\hline \multirow{2}{*}{ Chemical blend } & \multicolumn{2}{|c|}{$\begin{array}{c}\text { Mean catches }( \pm \text { SEM }) / \\
{\text { trap } / \text { day }^{\mathrm{b}}}\end{array}$} \\
\hline & Male & Female \\
\hline Synthetic sex pheromone $(\mathrm{Ph})$ & $138.4 \pm 4.9 \mathrm{~d}$ & $0.1 \pm 0.1 \mathrm{a}$ \\
\hline (Z)-3-Hexenyl acetate & $38.1 \pm 2.4 \mathrm{c}$ & $3.6 \pm 0.4 \mathrm{~cd}$ \\
\hline Allyl isothiocyanate & $25.8 \pm 3.4 \mathrm{~b}$ & $4.6 \pm 0.3 \mathrm{~d}$ \\
\hline (Z)-3-Hexen-1-ol & $34.9 \pm 2.2 \mathrm{c}$ & $1.7 \pm 0.4 \mathrm{~b}$ \\
\hline Benzaldehyde & $22.6 \pm 2.2 b$ & $2.1 \pm 0.4 \mathrm{~b}$ \\
\hline Phenylacetaldehyde & $25.4 \pm 2.3 \mathrm{~b}$ & $2.5 \pm 0.2 b$ \\
\hline Control & $2.4 \pm 0.4 \mathrm{a}$ & $0.2 \pm 0.1 \mathrm{a}$ \\
\hline
\end{tabular}

${ }^{a}$ Dosage of sex pheromone $(\mathrm{Ph})$ in lures was $50 \mu \mathrm{g}$, and dosage of each plant volatile was $5 \mu \mathrm{l}$ in $10 \mu \mathrm{l}$ peanut oil.

${ }^{b}$ Means within the same column followed by different letters are significantly different $(p<0.05$, Duncan's multiple range test). Analysis was carried out on transformed data $[\log (x+1)]$, and untransformed data are presented.

$F_{6,27}=78.01, \quad p<0.0001$; females: $F_{6,27}=12.57$, $p<0.0001$; Chinese cabbage field, males: $F_{6,41}=57.21, \quad p<0.0001 ;$ females: $F_{6,41}=27.11$, $p<0.0001$; cauliflower field, males: $F_{6,41}=13.01$, $p<0.0001$; females: $\left.F_{6,41}=5.44, p<0.0001\right)$ in the three brassicaceous crops tested in the field (DMRT, $p<0.05$ ) (Table 3). In comparison to traps 
Table 3. Average daily catches of male and female DBM by traps baited with synthetic sex pheromone in combination with plant volatiles in different crop fields ${ }^{\mathrm{a}}$

\begin{tabular}{|c|c|c|c|c|c|c|}
\hline \multirow{3}{*}{ Chemical blend } & \multicolumn{6}{|c|}{ Mean catches $( \pm$ SEM $) /$ trap $_{\text {day }}{ }^{\mathrm{b}}$} \\
\hline & \multicolumn{2}{|c|}{$\begin{array}{l}\text { Cabbage field } \\
\text { (May-July) }\end{array}$} & \multicolumn{2}{|c|}{$\begin{array}{l}\text { Chinese cabbage field } \\
\text { (October-December) }\end{array}$} & \multicolumn{2}{|c|}{$\begin{array}{l}\text { Cauliflower field } \\
\text { (May-July) }\end{array}$} \\
\hline & Male & Female & Male & Female & Male & Female \\
\hline Synthetic sex pheromone (Ph) & $98.3 \pm 5.3 b$ & $0.1 \pm 0.2 \mathrm{a}$ & $127.6 \pm 4.5 b$ & $0.3 \pm 0.3 \mathrm{a}$ & $13.2 \pm 3.3 \mathrm{~b}$ & $0.1 \pm 0.2 \mathrm{a}$ \\
\hline $\mathrm{Ph}+(Z)$-3-hexenyl acetate & $133.0 \pm 7.4 \mathrm{~d}$ & $5.8 \pm 0.8 \mathrm{c}$ & $240.9 \pm 6.2 c$ & $9.3 \pm 2.0 \mathrm{c}$ & $28.2 \pm 6.3 \mathrm{c}$ & $1.8 \pm 0.5 \mathrm{bc}$ \\
\hline $\mathrm{Ph}+$ allyl isothiocyanate & $93.0 \pm 7.3 \mathrm{~b}$ & $6.8 \pm 1.3 \mathrm{c}$ & $131.8 \pm 8.2 b$ & $11.3 \pm 1.5 \mathrm{c}$ & $16.7 \pm 5.1 \mathrm{~b}$ & $2.3 \pm 0.5 \mathrm{c}$ \\
\hline $\mathrm{Ph}+(Z)$-3-hexen-1-ol & $116.3 \pm 3.8 \mathrm{c}$ & $3.0 \pm 0.9 \mathrm{~b}$ & $232.2 \pm 5.6 \mathrm{c}$ & $9.0 \pm 1.2 \mathrm{c}$ & $26.5 \pm 7.4 \mathrm{c}$ & $1.5 \pm 0.3 \mathrm{bc}$ \\
\hline $\mathrm{Ph}+$ benzaldehyde & $103.0 \pm 4.2 \mathrm{bc}$ & $1.3 \pm 0.5 \mathrm{ab}$ & $134.2 \pm 4.8 b$ & $3.3 \pm 1.3 \mathrm{~b}$ & $12.3 \pm 1.2 \mathrm{~b}$ & $0.5 \pm 0.3 \mathrm{a}$ \\
\hline $\mathrm{Ph}+$ phenylacetaldehyde & $107.8 \pm 4.9 \mathrm{bc}$ & $1.5 \pm 0.6 \mathrm{ab}$ & $138.1 \pm 7.6 b$ & $4.7 \pm 1.5 b$ & $16.2 \pm 7.4 \mathrm{~b}$ & $1.0 \pm 0.4 \mathrm{ab}$ \\
\hline Control & $7.3 \pm 2.3 \mathrm{a}$ & $1.4 \pm 0.3 \mathrm{ab}$ & $6.9 \pm 3.8 \mathrm{a}$ & $1.0 \pm 1.5 \mathrm{a}$ & $2.2 \pm 1.4 \mathrm{a}$ & $1.0 \pm 0.4 \mathrm{ab}$ \\
\hline
\end{tabular}

${ }^{\text {a }}$ Dosage of sex pheromone $(\mathrm{Ph})$ in lures was $50 \mu \mathrm{g}$, and dosage of each plant volatile was $5 \mu \mathrm{l}$ in $10 \mu \mathrm{l}$ peanut oil.

${ }^{\mathrm{b}}$ Means within the same column followed by different letters are significantly different $(p<0.05$, Duncan's multiple range test).

Analysis was carried out on transformed data $[\log (x+1)]$, and untransformed data are presented.

Table 4. Average daily catches of male and female DBM by traps baited with synthetic sex pheromone in combination with plant volatiles in a cabbage field ${ }^{\mathrm{a}}$

\begin{tabular}{|c|c|c|}
\hline \multirow{2}{*}{ Chemical blend } & \multicolumn{2}{|c|}{ Mean catches $( \pm$ SEM $) /$ trap $_{\text {day }}{ }^{b}$} \\
\hline & Male & Female \\
\hline Synthetic sex pheromone $(\mathrm{Ph})$ & $151.3 \pm 9.3 b$ & $0.8 \pm 0.3 \mathrm{a}$ \\
\hline $\mathrm{Ph}+(Z)$-3-hexenyl acetate & $221.5 \pm 6.2 \mathrm{~d}$ & $15.8 \pm 2.2 \mathrm{c}$ \\
\hline $\mathrm{Ph}+(Z)-3$-hexenyl acetate + allyl isothiocyanate & $206.2 \pm 13.2 \mathrm{~cd}$ & $8.6 \pm 1.2 \mathrm{~b}$ \\
\hline $\mathrm{Ph}+(Z)-3-$ hexen-1-ol+allyl isothiocyanate & $193.3 \pm 6.3 \mathrm{c}$ & $14.2 \pm 1.3 \mathrm{c}$ \\
\hline $\mathrm{Ph}+(Z)$-3-hexenyl acetate $+(Z)$-3-hexen-1-ol + allyl isothiocyanate & $264.8 \pm 6.8 \mathrm{e}$ & $18.6 \pm 2.7 \mathrm{c}$ \\
\hline Control & $9.2 \pm 1.3 \mathrm{a}$ & $0.5 \pm 0.3 \mathrm{a}$ \\
\hline
\end{tabular}

${ }^{\text {a }}$ Dosage of sex pheromone $(\mathrm{Ph})$ in lures was $50 \mu \mathrm{g}$, and dosage of each plant volatile was $50 \mu \mathrm{l}$ in $10 \mu \mathrm{l}$ peanut oil.

${ }^{\mathrm{b}}$ Means within the same column followed by different letters are significantly different $(p<0.05$, Duncan's multiple range test).

Analysis was carried out on transformed data $[\log (x+1)]$, and untransformed data are presented.

baited with sex pheromone alone, traps baited with $\mathrm{Ph}+(Z)$-3-hexenyl acetate and $\mathrm{Ph}+(Z)$-3-hexen-1ol captured significantly more males and females in the Chinese cabbage field. Field trapping tests showed that females were caught in greater numbers when traps were baited with sex pheromone plus a single volatile compound. Very few females were caught by traps baited with sex pheromone alone and in control traps (Table 3).

Trapping efficiency of synthetic sex pheromone combined with mixed volatiles

Traps baited with synthetic sex pheromone $(\mathrm{Ph})$ in combination with mixed volatiles caught more males than those with synthetic sex pheromone alone (males: $F_{5,23}=371.74, p<0.0001$; females: $F_{5,23}=67.15, p<0.0001$ ) (Table 4). Traps baited with $\mathrm{Ph}+(Z)$-3-hexenyl acetate and $\mathrm{Ph}+(Z)-3$ hexenyl acetate $+(Z)$-3-hexen-1-ol+allyl isothiocyanate enhanced male catches by $46.4 \%$ and $75.0 \%$ respectively, compared with those with synthetic sex pheromone alone (DMRT, $p<0.05$ ) (Table 4). Traps baited with $\mathrm{Ph}+(Z)-3$-hexenyl acetate, $\mathrm{Ph}+(Z)$-3-hexen-1-ol+allyl isothiocyanate and $\mathrm{Ph}+(Z)$-3-hexenyl acetate $+(Z)-3$-hexen-1ol+allyl isothiocyanate caught an average of $15.8 \pm 2.2(6.7 \%$ females of all captured moths), $14.2 \pm 1.3(6.8 \%)$ and $18.6 \pm 2.7(6.6 \%)$ females/ trap/day, respectively, during the field trials. These three types of traps attracted significantly more females than those baited with $\mathrm{Ph}+(Z)$-3-hexenyl acetate + allyl isothiocyanate (Table 4 ). 


\section{DISCUSSION}

Plant volatiles are highly abundant in the plant kingdom and play an important role in plant-insect interactions (Visser, 1986). The composition of volatiles found in cabbage plants consists of fatty acid derivatives, terpenoids, benzenoids and a few sulfur-containing compounds, such as methyl isothiocyanate (Finch, 1978; Visser et al., 1979; Tollsten and Bergström, 1988; Reddy and Guerrero, 2004). In this study, the electrophysiological responses of DBM males and females to volatile components from Brassicaceae were tested in the laboratory. The EAG response results showed that benzaldehyde, phenylacetaldehyde and allyl isothiocyanate elicited stronger EAG responses than other components; however, in our field trapping tests, traps with sex pheromone in combination with benzaldehyde, phenylacetaldehyde or allyl isothiocyanate did not attract significantly more male moths than those with sex pheromone alone, pheromone $+(Z)-3$-hexenyl acetate, and pheromone $+(Z)-3$-hexen-1-ol in the three brassicaceous crops tested. Similar results were reported in Ips typographus (Zhang et al., 1999). Strong EAG responses have been recorded on antennae of male and female $I$. typographus when exposed to 1hexanol, (Z)-3-hexen-1-ol, and (E)-2-hexen-1-ol; however, a blend of the three volatiles reduced $I$. typographus trap catches by $85 \%$ compared to pheromone alone, while $41 \%$ to $50 \%$ reductions of trap catches were found when all three individual alcohols were added to the pheromone traps (Zhang et al., 1999). 1,4-Benzoquinone, the sex pheromone of Melolontha hippocastani, increased the number of male catches when combined at a ratio of $0.5: 5 \mathrm{mg}$ with $(Z)$-3-hexen-1-ol (Ruther and Hilker, 2003). (Z)-3-Hexenyl acetate significantly enhanced trap catches of Heliothis zea males when blended with the sex pheromone (Light et al., 1993). The same compounds may produce various effects in different insects. This variability may be enhanced when the habitat of the insect falls within a wide geographic range, suggesting the role of infochemicals in interactions under complex conditions.

It was also observed that more males were trapped with $(Z)$-3-hexenyl acetate than with allyl isothiocyanate, whereas more females were attracted with allyl isothiocyanate (Table 2). A possi- ble explanation for the field trapping results is that males may respond to host odors in order to locate host sites as well as an area of possible virgin female activity, and females may respond to host odors to locate or gain access to feeding and oviposition sites (Landolt and Phillips, 1997; Bruce et al., 2005). Furthermore, volatile chemicals produced by the hydrolysis of glucosinolates are also known to be involved in host plant location by crucifer specialists (Gupta and Thorsteinson, 1960; Renwick and Radke, 1990; Pivnick et al., 1994; Marazzi et al., 2004). Allyl isothiocyanates (also known as mustard oils), the hydrolysis products of mainly aliphatic glucosinolates, are feeding attractants for DBM and also stimulate/enhance oviposition (Reed et al., 1989; Justus and Mitchell, 1996; Hughes et al., 1997; Griffiths et al., 2001; Renwick et al., 2006).

Evidence presented in this study also indicates that traps baited with synthetic sex pheromone and some plant volatiles, such as ( $Z$ )-3-hexenyl acetate, enhanced male and female catches in comparison with traps baited with synthetic sex pheromone alone. These results are in accordance with those of previous studies in that $(Z)$-3-hexenyl acetate in combination with pheromone, in a $1: 1$ ratio, enhanced the number of females and males caught by traps in a cabbage field (Reddy and Guerrero, 2000 b). In this study, traps baited with pheromone $+(Z)-3$-hexenyl acetate and pheromone+ (Z)-3-hexenyl acetate $+(Z)-3$-hexen-1-ol + allyl isothiocyanate enhanced male catches by $46.4 \%$ and $75.0 \%$, respectively, compared with synthetic sex pheromone alone, and caught an average of $6.7 \%$ and $6.6 \%$ females of all captured moths, respectively (Table 4). This study reports that female diamondback moths were attracted to odorant chemicals from host plants and that there is a possible interaction between pheromones and kairomones in attracting males. This could not only help to understand pheromone-mediated mate finding and arresting behavior of DBM, but also may have important practical implications in the field utilization of plant volatiles as an approach to mass trapping in integrated pest management of DBM.

Meanwhile, the results from comparative trapping field tests of sex pheromone combined with mixed volatiles showed that the number of males trapped with pheromone $+(Z)$-3-hexenyl acetate + (Z)-3-hexen-1-ol+allyl isothiocyanate significantly 
increased compared with pheromone $+(Z)$-3-hexenyl acetate. Therefore, traps baited with pheromone $+(Z)$-3-hexenyl acetate $+(Z)-3$-hexen-1-ol+ allyl isothiocyanate would be a more effective combination for trapping male and female DBM. Further research will focus on the practical and economic formulation of pheromones in combination with plant volatiles in order to provide a stable release rate over a period of several weeks for use in field applications to control DBM by mass trapping.

\section{ACKNOWLEDGEMENTS}

We thank Prof. Jean-Marc Jallon, Prof. Yong-Ping Huang, Dr. Debbie Rae, and two anonymous reviewers for helpful comments. We also thank Dr. Hong-Yi Wei and Dr. Zhi-Wei Huang for their assistance in the field experiment. The research was funded by Science and Technology Projects of Shanghai and Guangdong Province (No. 023912004, 2007B020500002-5).

\section{REFERENCES}

Bruce, T. J. A., L. J. Wadhams and C. M. Woodcock (2005) Insect host location: a volatile situation. Trends Plant Sci. 10: 269-274.

Chow, Y. S., Y. M. Lin and C. L. Hsu (1977) Sex pheromone of the diamondback moth (Lepidoptera: Plutellidae). Bull. Inst. Zool. Acad. Sin. 16: 99-105.

Deng, J. Y., H. Y. Wei, Y. P. Huang and J. W. Du (2004) Enhancement of attraction to sex pheromones of Spodoptera exigua by volatile compounds produced by host plants. J. Chem. Ecol. 30: 2037-2045.

Dickens, J. C. (1984) Olfaction in the boll weevil Anthonomus grandis Boh. (Coleoptera: Curculionidae): electroantennogram studies. J. Chem. Ecol. 10: 1759-1785.

Dickens, J. C., J. W. Smith and D. M. Light (1993) Green leaf volatiles enhance sex attractant pheromone of the tobacco budworm, Heliothis virescens (Lepidoptera: Noctuidae). Chemoecology 4: 175-177.

Du, J. W. (2000) Current and future prospects for insect behavior-modifying chemicals in China. Agric. Chem. Biotechnol. 43: 222-229.

Duncan, D. B. (1955) Multiple range and multiple $F$ tests. Biometrics 11: 1-42.

Fang, Y. L. and Z. N. Zhang (2002) Influence of host-plant volatiles components on oviposition behavior and sex pheromone attractiveness to Helicoverpa armigera. Acta Entomol. Sin. 45: 63-67 (in Chinese with English summary).

Finch, S. (1978) Volatile plant chemicals and their effect on host plant finding by the cabbage root fly (Delia brassicae). Entomol. Exp. Appl. 24: 350-359.

Griffiths, D. W., N. Deighton, A. N. E. Birch, B. Patrian, R. Baur and E. Städler (2001) Identification of glucosinolates on the leaf surface of plants from the Cruciferae and other closely related species. Phytochemistry 57:
693-700.

Gupta, P. D. and A. J. Thorsteinson (1960) Food plant relationship of diamondback moth (Plutella maculipennis (Curt.)). II. Sensory relationship of oviposition of the adult female. Entomol. Exp. Appl. 3: 305-314.

Han, B. Y., Z. N. Zhang and Y. L. Fang (2001) Electrophysiology and behavior feedback of diamondback moth, Plutella xylostella, to volatile secondary metabolites emitted by Chinese cabbage. Chinese Sci. Bull. 46: 2086-2088.

Hughes, P. R., J. A. A. Renwick and K. D. Lopez (1997) New oviposition stimulants for the diamondback moth in cabbage. Entomol. Exp. Appl. 85: 281-283.

Justus, K. A. and B. K. Mitchell (1996) Oviposition site selection by diamondback moth, Plutella xylostella (L.) (Lepidoptera: Plutellidae). J. Insect Behav. 9: 887-898.

Koshihara, T. and H. Yamada (1980) Attractant activity of the female sex pheromone of diamondback moth, Plutella xylostella (L.), and analogue. Jpn. J. Appl. Entomol. Zool. 24: 6-12.

Koshihara, T., H. Yamada, Y. Tamaki and T. Ando (1978) Field attractiveness of the synthetic sex pheromone of the diamondback moth, Plutella xylostella (L.). Appl. Entomol. Zool. 13: 138-141.

Landolt, P. J. and T. W. Phillips (1997) Host plant influences on sex pheromone behavior of phytophagous insects. Annu. Rev. Entomol. 42: 371-391.

Light, D. M., R. A. Flath, R. G. Buttery, F. G. Zalom, R. E. Rice, J. C. Dickens and E. B. Jang (1993) Host plant green-leaf volatiles synergize the synthetic sex pheromones of the corn earworm and the codling moth (Lepidoptera). Chemoecology 4: 145-152.

Liu, Y. B. and B. E. Tabashnik (1997) Visual determination of sex of diamondback moth larvae. Can. Entomol. 129: 585-586.

Marazzi, C., B. Patrian and E. Städler (2004) Secondary metabolites of the leaf surface affected by sulfur fertilization and perceived by the diamondback moth. Chemoecology 14: 81-86.

Môttus, E., V. Nômm, I. H. Williams and I. Liblikas (1997) Optimization of pheromone dispensers for diamondback moth Plutella xylostella. J. Chem. Ecol. 23: 2145-2159.

Pivnick, K. A., J. J. Blair and G. P. Slater (1994) Identification of olfactory cues used in host-plant finding by diamondback moth, Plutella xylostella (Lepidoptera: Plutellidae). J. Chem. Ecol. 20: 1407-1427.

Reddy, G. V. P. and A. Guerrero (2000a) Pheromone-based integrated pest management to control the diamondback moth Plutella xylostella in cabbage fields. Pest Manag. Sci. 56: 882-888.

Reddy, G. V. P. and A. Guerrero (2000b) Behavioral responses of the diamondback moth to green leaf volatiles of Brassica oleracea subsp. capitata. J. Agric. Food Chem. 48: 6025-6029.

Reddy, G. V. P. and A. Guerrero (2004) Interactions of insect pheromones and plant semiochemicals. Trends Plant Sci. 9: 253-261.

Reed, D. W., K. A. Pivnick and E. W. Underhill (1989) Identification of chemical oviposition stimulants for the dia- 
mondback moth, Plutella xylostella, present in 3 species of Brassicaceae. Entomol. Exp. Appl. 53: 277-286.

Renwick, J. A. A. and C. D. Radke (1990) Plant constituents mediating oviposition by the diamondback moth, Plutella xylostella (L.) (Lepidoptera: Plutellidae). Phytophaga 3: $37-46$.

Renwick, J. A. A., M. Haribal, S. Gouinguené and E. Städler (2006) Isothiocyanates stimulating oviposition by the diamondback moth, Plutella xylostella. J. Chem. Ecol. 32: $755-766$.

Ruther, J. and M. Hilker (2003) Attraction of forest cockchafer Melolontha hippocastani to (Z)-3-hexen-1-ol and 1,4-benzoquinone: application aspects. Entomol. Exp. Appl. 107: 141-147.

Talekar, N. S. and A. M. Shelton (1993) Biology, ecology, and management of the diamondback moth. Annu. Rev. Entomol. 38: 275-301.

Tamaki, Y., K. Kawasaki, H. Yamada, T. Koshihara, N. Osaki, T. Ando, S. Yoshida and H. Kakinohana (1977) Z-11-
Hexadecenal and Z-11-hexadecenyl acetate: Sexpheromone components of the diamondback moth (Lepidoptera: Plutellidae). Appl. Entomol. Zool. 12: 208-210.

Tollsten, L. and G. Bergström (1988) Headspace volatiles of whole plants and macerated plant parts of Brassica and Sinapis. Phytochemistry 27: 2073-2077.

Visser, J. H. (1986) Host odour perception in phytophagous insects. Annu. Rev. Entomol. 31: 121-144.

Visser, J. H., S. Van Straten and H. Maarse (1979) Isolation and identification of volatiles in the foliage of potato, Solanum tuberosum, a host plant of the Colorado potato beetle, Leptinotarsa decemlineata. J. Chem. Ecol. 5: $13-25$.

Wang, B. R. (1987) Organic Synthesis. Science Press, Beijing. 785 pp. (in Chinese with English summary).

Zhang, Q. H., F. Schlyter and P. Anderson (1999) Green leaf volatiles interrupt pheromone response of spruce bark beetle, Ips typographus. J. Chem. Ecol. 25: 2847-2861. 